\section{Influencia del tiempo pos aclaramiento sobre la fuerza de adhesión al esmalte}

\section{Influence of post-bleaching time on enamel bond strength}

\section{Resumen}

El intervalo de tiempo trascurrido pos aclaramiento dentario es una variable significativa para la adhesión a la estructura dentaria. Los agentes aclaradores utilizados sobre la superficie del esmalte, debido a la degradación de los peróxidos empleados generan radicales libres de oxígeno que alteran la fuerza de adhesión. La literatura ha demostrado que el tiempo trascurrido desde un aclaramiento hasta el momento de la restauración es determinante para la adhesión al esmalte. El objetivo de este trabajo fue hacer una revisión de literatura sobre la influencia del tiempo pos aclaramiento dental y su efecto en la fuerza de adhesión al esmalte dental de las resinas compuestas. Para la recopilación de datos se utilizaron las siguientes bases de datos: SciELO, EBSCO y repositorios académicos, obteniéndose 36 artículos y tesis. Después del análisis, se puede considerar que el tiempo es determinante en la resistencia adhesiva y que las alteraciones posteriores al aclaramiento dentario se extienden hasta la semana posterior prolongándose inclusive hasta cuatro semanas, tiempo que la literatura estima necesario para la eliminación del oxígeno residual.

Palabras clave: Aclaramiento dental; Adhesión dental; Peróxidos; Antioxidantes (fuente: DeCS BIREME).

\begin{abstract}
The time interval after tooth bleaching is a significant variable for the adhesion to the tooth structure. The bleaching agents used on the enamel surface, due to the degradation of the peroxides used, generate oxygen free radicals that alter the bonding strength. The literature has shown conclusively that the time that has elapsed since a bleaching to the moment of restoration is decisive for the adhesion to the enamel. The objective of this work was to review the literature about the influence of time after dental bleaching and its effect on the strength of adhesion to enamel of composite resins. Data collection was carried out through electronic databases such as: Scielo, EBSCO, academic repositories, obtaining 36 articles and thesis. After the analysis, it can be considered that time is decisive in the adhesive resistance and that the alterations after tooth bleaching extend until the subsequent week, lasting up to four weeks, a time that the literature considers necessary for the elimination of residual oxygen.
\end{abstract}

Keywords: Tooth bleaching; Dental bonding; Peroxides; Antioxidants (source: $\mathrm{MeSH}$ NLM).
ISSN-L 1560-9111; eISSN: 1609-8617

\section{Artículo de Revisión}

Nilda María del Rosario Alvarez ${ }^{1, a}$, Juan José Christiani ${ }^{1, b}$, María Natalia Mandri ${ }^{1, b}$

${ }^{1}$ Universidad Nacional del Nordeste, Facultad de Odontología, Corrientes, Argentina.

a Magister en Salud Bucal.

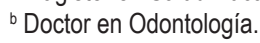

\section{Correspondencia:}

Nilda María del Rosario Alvarez: nalvarez@odn.unne. edu.ar

Facultad de Odontología UNNE. Av. Libertad 5450.

Corrientes, Argentina.

ORCID: 0000-0003-3664-2155

\section{Coautores:}

Juan José Christiani: jjchristiani@odn.unne.edu.ar ORCID: 0000-0002-8627-9174

María Natalia Mandri: nataliamandri@hotmail.com ORCID: 0000-0001-6312-7242

\section{Editora:}

Rosse Mary Falcón-Antenucci

Universidad Inca Garcilaso de la Vega, Perú.

Conflicto de intereses: los autores declaran no tener conflictos de interés.

Fuente de financiamiento: ninguna.

Recibido: 06/07/20

Aceptado: 10/11/20

Publicado: $15 / 02 / 21$ 


\section{Introducción}

El aclaramiento dental es uno de los procedimientos odontológicos más utilizados en la actualidad, generalmente precede a tratamientos restauradores, rehabilitadores y ortodóncicos ${ }^{1}$. Las sustancias usadas para aclarar actúan como potentes oxidantes, mediante la formación de radicales libres, moléculas de oxígeno reactivas y aniones de peróxido de hidrógeno que penetran en el esmalte y la dentina atacando a las macromoléculas orgánicas altamente cromógenas, responsables por la descoloración. Al descomponerlas, los anillos carbonados pasan a convertirse en cadenas lineales con dobles enlaces, aún cromáticas. Si el proceso persiste, los radicales libres terminan rompiendo los dobles enlaces y las cadenas carbonadas resultantes, ya con enlaces simples se hacen incoloras. Siendo el resultado el aclaramiento del color del diente ${ }^{2}$.

Los químicos utilizados para realizar los tratamientos de aclaramiento son peróxidos, que son compuestos obtenidos de la reacción entre un óxido con el oxígeno monoatómico. Por lo tanto, un peróxido es un óxido que dispone de un nivel más alto de oxígeno que los óxidos corrientes ${ }^{3-5}$. Generalmente los peróxidos utilizados para la técnica de aclaramiento son los de hidrógeno y de carbamida en diferentes concentraciones. El peróxido de hidrógeno, también conocido como agua oxigenada, dioxogen o dioxidano es conocido por ser un poderoso oxidante. Mientras que el peróxido de carbamida es un peróxido de hidrógeno con úrea, que es un compuesto orgánico ${ }^{3-5}$.

Diversos estudios ${ }^{3-5}$ han reportado reducciones significativas en la resistencia a la adhesión de las restauraciones con resinas compuesta al esmalte dentario aclarado. Estas fallas adhesivas están en relación directa con el intervalo de tiempo pos aclaramiento, con el tipo de peróxido (de hidrógeno o de carbamida) y las concentraciones de estos ${ }^{6,7}$. Algunos autores señalan que antes de realizar cualquier procedimiento adhesivo se deben inhibir los radicales libres de oxígeno con el uso de antioxidantes ${ }^{8-11}$, o esperar un intervalo igual o mayor a siete días ${ }^{12,13}$. Debido a que, se producen cambios en la microestructura del esmalte sometido al proceso de aclaramiento ${ }^{4-12}$. Entretanto, otros estudios indican que los peróxidos no alteran la estructura morfológica del esmalte ${ }^{14}$.

Aún existen controversia en relación a la influencia del aclaramiento dental en la resistencia adhesiva de las resinas compuestas al esmalte ${ }^{12,14}$, siendo que, es un tratamiento realizado con frecuencia en la práctica clínica. Es fundamental que el cirujano-dentista conozca los efectos sobre la adhesión en restauraciones realizadas poco tiempo después de un aclaramiento dental. Por lo tanto, el objetivo de este artículo es presentar una revisión sobre la influencia del tiempo pos aclaramiento dental y su relación con la fuerza de adhesión al esmalte dental, señalando los factores directos que influencian en las fallas adhesivas.

\section{Revisión de la literatura}

El agente aclarador más utilizado en los consultorios odontológicos es el peróxido de hidrógeno en altas concentraciones, debido a que ofrece mejores resultados, seguido de los blanqueadores a base de peróxido de carbamida que presentan una acción más lenta y con más riesgos de irritación de las encías y deshidratación de las estructuras dentarias por ser un gel anhídrido ${ }^{15}$.

Mc Guckin et al. ${ }^{16}$ y Titley et al. ${ }^{17}$ reportaron que la presencia de peróxido residual, oxígeno liberado por las sustancias utilizadas para el aclaramiento dentario, inhibe considerablemente la adhesión de la resina compuesta al esmalte dentario.

\section{Intervalo de tiempo trascurrido}

Estudios ${ }^{14,15}$ muestran fallas adhesivas en la interface esmalte/resina compuesta, pos aclaramiento dentario; situando al intervalo de tiempo transcurrido entre el aclaramiento y la restauración con resina como una de las variables fundamentales. Debido al tiempo de permanencia de los radicales libres en los tejidos dentarios. En esos estudios, el tiempo varió de una hasta cuatro semanas posteriores al procedimiento restaurador.

Para contrarrestar esos radicales libres, muchos trabajos de investigación ${ }^{1,8-11}$ reportan el uso de antioxidantes como: catalasa o ascorbato sódico para inactivar los radicales libres del oxígeno residual, revertir las consecuencias fisicoquímicas indeseables sobre la estructura dental y mejorar la adhesión al sustrato adamantino.

\section{Concentración de los agentes aclaradores}

Tezel et al. ${ }^{18}$, señalan que los valores de reducción en las fuerzas adhesivas están en relación directa con las concentraciones de los peróxidos utilizados. Lo que también fue verificado por Borges et al. ${ }^{19}$, que evaluaron la cantidad de oxígeno residual, comparando dos peróxidos: peróxido de hidrógeno al 35\% y peróxido de carbamida al 37\%. Los autores concluyeron que el peróxido de carbamida al 37\% fue el que más radicales libres dejaba, por lo tanto, la fuerza de adhesión en dientes aclarados con este peróxido era menor.

Resultados opuestos fueron encontrados por Carvalli et al. ${ }^{20}$ al no obtener diferencias significativas entre las concentraciones de los agentes aclaradores y su influencia en la resistencia a la tracción. Al ser evaluado diferentes concentraciones de peróxido de carbamina, utilizando el test de microtensión a $0,5 \mathrm{~mm} / \mathrm{min}$ en molares (6 grupos de 10) sometidos a diferentes agentes aclaradores: Opalescence al 10\%, 15\%, 20\%, y Whiteness al $10 \%$ y $16 \%$. También fue observado que, las muestras sometidas a aclaramiento mostraron menor fuerza de adhesión que el grupo control.

De la misma manera, otro estudio ${ }^{21}$ refuerza la idea que el peróxido de carbamida independientemente de la concentración utilizada, produce menores valores de resistencia a la fuerza de adhesión. Esto fue verificado al evaluar la resistencia de adhesión, después del aclaramiento dentario con peróxido de carbamida al 10\% y peróxido de hidrógeno al 35\%. A pesar que la concentración es mayor en el segundo grupo, los niveles más bajos de adhesión lo obtuvieron con el primero. 
Concordando con la idea anterior Quispe ${ }^{22}$, verificó que el uso de aclaradores dentales disminuye considerablemente la fuerza de adhesion, independentemente del porcentaje de concentración del mismo. Esta investigacion evaluó in vitro la fuerza de adhesión de brackets en dientes aclarados con peróxido de hidrógeno al 35\% y peróxido de carbamida al 10\%, en premolares humanos divididos en tres grupos: I (control) sin aclaramiento, II con peróxido de hidrógeno al 35\%, III con peróxido de carbamida al $10 \%$. Los resultados mostraron que no existe diferencia estadísticamente significativa entre los tres grupos.

\section{Tiempo pos aclaramiento dentario}

La variable tiempo pos aclaramiento dentario es fundamental para obtener elevados valores de adhesión al esmalte dentario. García-Godoy et al. ${ }^{23}$ y Ben-Amar et al. ${ }^{24}$ señalan que los cambios estructurales y morfológicos producidos por los peróxidos persisten horas, días hasta inclusive semanas, atribuyendo esta consecuencia a los bajos valores de adhesión.

A medida que pasa el tiempo pos aclaramiento, los valores de adhesión aumentan, como fue reportado por Unlu et al. ${ }^{7}$ al evaluar la resistencia de unión de Clearfil SE Bond y Clearfil ST, en dientes sometidos a aclaramiento con peróxido de carbamida $10 \%$ y peróxido de hidrógeno $35 \%$. La medición fue realizada: inmediatamente, 24 horas, 1 semana y 2 semanas después. Los resultados demostraron que los valores inmediatos decrecieron significativamente después de 24 horas y que los valores obtenidos en los grupos de 1 y 2 semanas eran semejantes al grupo control.

Sundfeld et al. ${ }^{13}$, evaluaron la fuerza adhesiva del esmalte pos aclaramiento con peróxido de hidrógeno al 35\%, en 4 tiempos experimentales: inmediatamente, a los 7 , 14 y 21 días. Señalaron que, 7 días es el tiempo necesario para obtener resultados similares a los del grupo control (sin aclaramiento). En discrepancia con este autor emergieron estudios como los de Homewood et al. ${ }^{25}$ que señalan que después de 24 horas del aclaramiento los valores de fuerzas de adhesión no se ven afectados. Van der Vyver et al. ${ }^{12}$ reportaron que las variaciones en los valores de adhesión se mantienen inclusive hasta cuatro semanas después de realizado en tratamiento de aclaramiento dentario. Asimismo, señalan que la fuerza de adhesión es paulatina, con una recuperación a las dos semanas del 76,93\%.

Halil et al. ${ }^{26}$ consideran fundamental retrasar 14 días la realización de técnicas adhesivas o bien realizar la aplicación de ascorbato de sodio como antioxidante, lo cual permitiría revertir el efecto del oxígeno residual adelantando una semana el tratamiento con técnicas adhesivas.

Moosavi et al. ${ }^{27}$ evaluaron la influencia del uso de diferentes agentes aclaradores de peróxido de hidrógeno en la adhesión de resina compuesta unida al esmalte aclarado a través del test de cizallamiento. Manipularon premolares divididos en cuatro grupos: grupo 1 aclarado con gel de peróxido de hidrógeno al $35 \%$; grupo
2: aclarado con 35\% de gel de peróxido de hidrógeno contenido caseína fosfopéptido-amorfo fosfato de calcio (CPP-ACP); grupo 3: aclarado con hidrógeno al 35\% gel de peróxido combinado con fluoruro; y grupo 4: aclarado con 35\% peróxido de hidrógeno aplicando una semana antes de la colocación de restauración de resina compuesta. Después de 24 horas de almacenamiento y 1000 ciclos de termociclado, procedieron a realizar test de cizallamiento midiendo la fuerza de unión al corte de las muestras, con una velocidad de cruceta de 0,5 $\mathrm{mn} / \mathrm{min}$. Señalaron que el uso de sustancias aclaradoras disminuyó la fuerza de adhesión de la resina compuesta al esmalte cuando se usó inmediatamente después del aclaramiento.

Totoy ${ }^{28}$, evaluó in vitro la influencia del tiempo pos aclaramiento con peróxido de carbamida al $22 \%$ sobre la adhesión de una resina compuesta al esmalte dental. Indicando que el tiempo pos aclaramiento para lograr una fuerza de adhesión es primordial, en contraposición con otros autores remarca la necesidad de esperar más de tres semanas para realizar técnicas adhesivas. Para ello analizó premolares divididos en 6 grupos, uno control (sin aclaramiento), y en los demás grupos aplicó el agente aclarador para después aplicar la técnica adhesiva, en el primer grupo en forma inmediata, en el segundo grupo a las $24 \mathrm{~h}$ y así sucesivamente en los otros grupos a los 7, 14 y 28 días. Todos los grupos fueron sometidos a pruebas de tracción, concluyendo que el éxito para una buena adhesión entre una resina y un diente pos aclarado está en función del tiempo, los valores más altos de adhesión en comparación con el grupo control lo obtuvieron a los 28 días.

Bittencourt et al. ${ }^{29}$ evaluaron la fuerza de adhesion a través del test de cizallamiento de restauraciones con composite, teniendo en cuenta intervalos de tiempo transcurrido pos aclaramiento dental con peróxido de hidrógeno al 35\%. Concluyeron que el tratamiento reduce la fuerza de adhesión al esmalte, para lo cual es necesario esperar siete días antes de realizar procedimientos de restauración con técnicas adhesivas.

Baldion Elorza ${ }^{1}$, analizó la Influencia del tiempo pos aclaramiento con peroxido de hidrogeno sobre la adhesión de una resina compuesta al esmalte dental. Para ello los especimenes fueron divididos en 6 grupos: un grupo control al que solo se le practicó la técnica adhesiva y cinco grupos de estudios a los cuales se les aplicó el agente para aclaramiento. Las técnicas adhesivas fueron realizadas en diferentes intervalos de tiempo: $0,1,7,14$ y 28 días. Los valores de adhesión en los grupos con aclaramiento de los 0 a los 28 días fueron estadísticamente menores al grupo control. Concluyendo que el peróxido de hidrógeno reduce los valores de adhesión al esmalte dental, asimismo demostró que el tiempo pos aclaramiento es determinante para recuperar la resistencia de unión de la resina compuesta.

Zarate et al. ${ }^{30}$, comparó la fuerza de adhesión al esmalte de piezas blanqueadas con ozono y con peróxido de carbamida. La medición se realizó con un microtensiómetro a una velocidad de $0.5 \mathrm{~mm} / \mathrm{min}$ hasta su fractura. 
Se dividieron en tres grupos: control (saliva) y dos experimentales (peróxido de carbamida y ozono). Los grupos experimentales fueron expuestos a la aplicación de los agentes aclaradores y se realizó una restauración con resina a los $0,1,7,14$, y 21 días pos aclaramiento. No hubo diferencia estadísticamente significativa en 0 y 1 días pos aclaramiento en ambos grupos, la fuerza de adhesión del grupo con ozono comparada con el grupo control fue similar y los grupos tratados con peróxido de carbamida mantuvieron valores bajos de adhesión hasta 3 semanas después del aclaramiento.

Gutiérrez ${ }^{31}$ realizó un estudio in vitro comparando la influencia del tiempo pos aclaramiento dental sobre la adhesión de una resina al esmalte dental. Utilizó peróxido de hidrógeno al $38 \%$, esperando en uno de los grupos 7 días y en otro 15 días. Concluyó que el tiempo de espera es un factor importante para mejorar la fuerza adhesiva, los mejores valores de adhesión fueron encontrados a los 15 días pos aclaramiento.

Monteiro et al. ${ }^{32}$ evaluaron la fuerza de adhesión (esmalte-restauración a base de silorano) a través de test de cizallamiento de piezas dentarias blanqueadas. Se utilizaron cuatro grupos, el grupo uno y dos denominados control (sin aclaramiento) y los grupos tres y cuatro experimentales, estos últimos fueron sometidos a tratamiento con peróxido de hidrógeno al 38\%. El análisis comparativo demostró diferencias significativas después del aclaramiento para la unión adhesiva de los materiales de restauración a base de metacrilato, no así para los materiales compuestos a base de siloranos.

Un estudio realizado por Solís et al. ${ }^{33}$ evaluó la fuerza de adhesión de resinas compuestas, al esmalte dental pos aclaramiento con peróxido de carbamida al 15\%. Sesenta premolares fueron divididos en cinco grupos: un grupo control en el que realizó la adhesión sin aclaramiento, y cuatro restantes a los cuales se aplicó el agente aclarador. El intervalos de tiempo posterior al aclaramiento fue: 24 horas, 7, 14 y 21 días. Los resultados mostraron que el peróxido de carbamida al 15\% reduce significativamente la fuerza adhesiva, y la fuerza de adhesión aumenta conforme aumenta el tiempo pos aclaramiento.

Sanchez Tito et al. ${ }^{34}$ evaluaron el efecto del peróxido de hidrógeno al $40 \%$ sobre la fuerza de adhesión de brackets metálicos cementados en distintos intervalos de tiempo. Se cementaron los brackets en diferentes intervalos de tiempo pos aclaramiento: grupo I - 1 hora después, grupo II - a los 7 días, grupo III a los 14 días, grupo IV control. Se midió la fuerza de adhesión de brackets a través del test de cizallamiento. Los resultados demostraron que el aclaramiento dental reduce la fuerza de adhesión, los grupos I y II presentaron valores bajos en la fuerza de adhesión mientras que el grupo III fue similar al grupo control. Lo cual remarca que el intervalo de tiempo transcurrido pos aclaramiento es determinante en relación a la fuerza de adhesión. Señalaron que el cementado de brackets debe ser realizado posterior a las dos semanas, tiempo en que los valores de adhesión registrados son similares al grupo sin aclaramiento. Por otra parte Peña ${ }^{35}$, indica que los valores de resistencia adhesiva aumentan recien a las tres semanas de haberse realizado el aclaramiento dentario. Para ello midió la fuerza adhesiva al esmalte dentario, por medio del test de cizallamiento, se utilizaron premolares humanos divididos en tres grupos: control, grupo con aclaramiento con peróxido de hidrógeno al 38\% y el otro con peróxido de carbamida al 45\%. La resina aplicada (Filtek Z350, 3M-ESPE, MN, USA) fue evaluada a las 24 horas y a las 3 semanas. Los especímenes sometidos a claramiento tanto con peróxido de hidrógeno al 38\% o de carbamida al $45 \%$ presentaban una reducción de la fuerza de adhesión al esmalte después de un día, mientras que Criollo ${ }^{36}$, refuerza la importancia del intervalo de tiempo al obtener mejor adhesión en el periodo más prolongado de tiempo (20 días). En su estudio analizó la fuerza de adhesión sobre el esmalte de diente bovino, para esto se utilizó peróxido de hidrógeno al $40 \%$ y peróxido de carbamida al 20\%. Los intervalos de tiempo fueron de: 1, 10, y 20 días. Se analizaron 3 grupos: grupo control y dos grupos experimentales, uno de ellos tratados con peróxido de carbamida al $20 \%$ y el otro con peróxido de hidrógeno al $40 \%$; y la fuerza de adhesión fue medida a las 24 horas, 10 días, y 20 días pos aclaramiento.

\section{Conclusiones}

- Es fundamental que el profesional odontólogo maneje ciertas técnicas y tiempos necesarios para reducir al máximo los efectos indeseables de los agentes aclaradores sobre el esmalte dentario.

- La mayoría de los estudios indican que la concentración de los agentes aclaradores no es un factor determinante sobre la fuerza adhesiva de resinas reforzadas al esmalte dentario.

- El tiempo transcurrido es un factor importante sobre la fuerza de adhesión, así las mayores fallas se observaron dentro de las 24 horas hasta los 7 dias pos aclaramiento. El tiempo ideal de espera para realizar un tratamiento restaurador seria de dos a tres semanas pos aclaramiento.

\section{Referencias bibliográficas}

1. Baldión Elorza PA. Influencia del tiempo posblanqueamiento sobre la adhesión de una resina compuesta al esmalte dental. Rev Fac Odontol Univ Antioq. 2013;25(1):92-116.

2. Minoux M, Serfaty R. Vital tooth bleaching: biologic adverse effects-a review. Quintessence Int. 2008;39(8):64559.

3. Lai SCN, Tay FR, Cheung GSP, Mak YF, Carvalho RM, Wei SHY, Toledano M, Osorio R, Pashley DH. Reversal of compromised bonding in bleached enamel. J Dent Res. 2002;81(7):477-81. DOI: $10.1177 / 154405910208100709$.

4. Cimilli H, Pameijer $\mathrm{CH}$. Effect of carbamide peroxide bleaching agents on the physical properties and chemical composition of enamel. Am J Dent. 2001 [Citado el 14 de marzo de 2020];14(2):63-6. Disponible en: https:// pubmed.ncbi.nlm.nih.gov/11507801/. 
5. Dishman MV, Covey DA, Baughan LW. The effects of peroxide bleaching on composite to enamel bond strength. Dent Mater. 1994;10(1):33-6. DOI: 10.1016/01095641(94)90019-1.

6. Borges GA, Pedreira GA, Martinelli J, Oliveira WJ. A influência do clareamento dental na resistência de união na interfase resina-esmalte. ROBRAC 2006;15(40):46-54.

7. Unlu N, Cobankara FK, Ozer F. Effect of elapsed time following bleaching on the shear bond strength of composite resin to enamel. J Biomed Mater Res B Appl Biomater. 2008;84(2):363-8. DOI: 10.1002/jbm.b.30879.

8. Rotstein I. Role of catalase in the elimination of residual hydrogen peroxide following tooth bleaching. J Endod. 1993;19(11):567-9. DOI: 10.1016/S00992399(06)81288-4.

9. Türkün M, Kaya AD. Effect of $10 \%$ sodium ascorbate on the shear bond strength of composite resin to bleached bovine enamel. J Oral Rehabil. 2004;31(12):118491. DOI: $10.1111 /$ j.1365-2842.2004.01369.x.

10. Attin T, Muller T, Patyk A, Lennon AM. Influence of different bleaching systems on fracture toughness and hardness of enamel. Oper Dent. 2004;29(2):188-95.

11. Türkün M, Celik EU, Kaya AD, Arici M. Can the hydrogel form of sodium ascorbate be used to reverse compromised bond strength after bleaching? J Adhes Dent. 2009;11(1):35-40.

12. van der Vyver PJ, Lewis SB, Marais JT. The effect of bleaching agent on composite/enamel bonding. J Dent Assoc S Afr. 1997;52(10):601-3.

13. Sundfeld RH, Briso AL, De Sá PM, Sundfeld ML, Bedran-Russo AK. Effect of time interval between bleaching and bonding on tag formation. Bull Tokyo Dent Coll. 2005;46(1-2):1-6. DOI: 10.2209/tdcpublication.46.1.

14. Berga Caballero A, Forner Navarro L, Amengual Lorenzo J. In vivo evaluation of the effects of $10 \%$ carbamide peroxide and 3.5\% hydrogen peroxide on the enamel surface. Med Oral Patol Oral Cir Bucal. 2007;12(5):E404-7.

15. Kwon SR. Whitening the single discolored tooth. Dent Clin North Am. 2011;55(2):229-39. DOI: 10.1016/j. cden.2011.01.001.

16. Mc Guckin RS, Thurmond BA, Osovitz S. Enamel shear bond strengths after vital bleaching. Am J Dent. 1992;5(4):216-22.

17. Titley KC, Torneck CD, Smith DC, Chernecky R, Adibfar A. Scanning electron microscopy observations on the penetration and structure of resin tags in bleached and unbleached bovine enamel. J Endod. 1991;17(2):72-5. DOI: 10.1016/S0099-2399(06)81611-0.

18. Tezel H, Ertaş OS, Ozata F, Dalgar H, Korkut ZO. Effect of bleaching agents on calcium loss from the enamel surface. Quintessence Int. 2007;38(4):339-47.

19. Borges GA, Pedreira GA, Martinelli J, Oliveira WJ. A influência do clareamento dental na resistência de união na interfase resina-esmalte. ROBRAC 2006;15(40):46-54.

20. Cavalli V, Giannini M, Carvalho RM. Effect of carbamide peroxide bleaching agents on tensile strength of human enamel. Dent Mater. 2004;20(8):733-9. DOI: 10.1016/j.dental.2003.10.007.
21. Marson FC, Sensi LG, Arruda T. Efeito do clareamento dental sobre a resistência adesiva do esmalte. RGO. 2008;56(1):33-7.

22. Quispe AA. Comparación in vitro de la fuerza de adhesion de brackets sobre dientes con y sin blanqueamiento. [Tesis de grado] Facultad de Medicina: Estomatología, Universidad Privada Antenor Orrego; 2014. 60p.

23. García-Godoy F, Dodge WW, Donohue M, O’Quinn JA. Composite resin bond strength after enamel bleaching. Oper Dent. 1993;18(4):144-7.

24. Ben-Amar A, Liberman R, Gorfil C, Bernstein Y. Effect of mouthguard bleaching on enamel surface. Am J Dent. 1995;8(1):29-32.

25. Homewood C, Tyas M, Woods M. Bonding to previously bleached teeth. Aust Orthod J. 2001;17(1):27-34.

26. Kılınç Hİ, Aslan T, Kılıç K, Er Ö, Kurt G. Effect of delayed bonding and antioxidant application on the bond strength to enamel after internal bleaching. J Prosthodont. 2016;25(5):386-91. DOI: 10.1111/jopr.12303.

27. Moosavi H, Moghaddas MJ, Ghoddusi J, Rajabi O. Effects of two antioxidants on the microleakage of resin-based composite restorations after nonvital bleaching. J Contemp Dent Pract. 2010;11(6):E033-40.

28. Totoy CFJ. Estudio in vitro de la influencia del tiempo postblanqueamiento realizado con peróxido de carbamida al 22\% sobre la adhesión de una resina compuesta al esmalte dental en dientes premolares. [Tesis de grado]. [Quito]. Facultad de Odontología. Universidad Nacional de Ecuador. Quito; 2017. 83p. http://www.dspace. uce.edu.ec/handle/25000/8354.

29. Bittencourt ME, Trentin MS, Linden MS, de Oliveira Lima Arsati YB, França FM, Flório FM, Basting RT. Influence of in Situ postbleaching times on shear bond strength of resin-based composite restorations. J Am Dent Assoc. 2010;141(3):300-6. DOI: 10.14219/jada. archive.2010.0164.

30. Zarate AM, Bermejo G, Bazan JPL, Saravia M. Efectos de un blanqueamiento dental con ozono y otro con peróxido de carbamida al $22 \%$ sobre la fuerza de adhesión al esmalte en diferentes intervalos de tiempo. Acta Odontol Venez. [Internet]. 2009 [citado el 10 de junio de 2020]; 47(4):69-77. Disponible en: http://ve.scielo.org/scielo.php?script=sci_arttext\&pi$\mathrm{d}=$ S000163652009000400007\&lng=es.

31. Gutiérrez LMC. Análisis comparativo de la influencia del tiempo post aclaramiento dental sobre la adhesión de una resina compuesta al esmalte dental. Estudio in-vitro. [Tesis de Bachiller] [Quito] Facultad de Odontología. Universidad de las Américas; 2016. 71p.

32. Monteiro TL, Carneiro NCM, Nogueira BCL, Braga EMF, Souza Júnior MHS, Loretto SC. Influence of tooth bleaching on the bonding strength of Silorane-based restorative system. Int J Odontostomat. 2015;9(3):497504. DOI: 10.4067/S0718-381X2015000300023.

33. Solís MZ, Illicachi AK. Evaluación de la fuerza de adhesión de resinas compuestas con relación al tiempo, en esmalte dental postblanqueado con peróxido de carbamida al 15\%, estudio in vitro. [Tesis de bachiller] [Quito] Facultad de Odontología. Universidad Nacional de Ecuador; 2016. 78p. 
34. Sánchez Tito MA, Kuong NG. Efecto del peróxido de hidrógeno al $40 \%$ sobre la fuerza de adhesión de brackets metálicos. Rev Estomatol Herediana. [online]. 2017 [citado el 14 abril de 2020];27(2):81-7. Disponible en: http://www.scielo.org.pe/scielo.php?pi$\mathrm{d}=\$ 101943552017000200004 \&$ script=sci_arttext.

35. Peña MC. Resistencia al cizallamiento de una resina compuesta al aplicar diferentes agentes blanqueadores y su efecto sobre la microestructura del esmalte. [Tesis Doctoral]. [Sevilla]: Universidad de Sevilla. 2014; 130p. Disponible en: https://idus.us.es/bitstream/handle/11441/65510/S_TD_PROV130.pdf?sequence=5\&isAllowed $=y$.
36. Criollo LEM. Fuerzas de adhesión en esmalte, post clareamiento dental con peróxido de hidrogeno al 40\% y peróxido de carbamida al 20\%, en intervalos de tiempo de 1, 10, y 20 días. Estudio in vitro. [Tesis de grado]. [Quito]: Facultad de Odontología, Universidad Nacional de Ecuador 2017. 59 p. http://www.dspace.uce.edu. ec/handle/25000/8331. 